\title{
Pharmacokinetics in anaesthesia
}

J.A. Moffat PH D, B. Milne MD

\section{CONTENTS}

\section{Pharmacokinetics in Anaesthesia \\ Introduction}
(a) Background
(b) History of pharmacokinetics
Definitions
Aging and pharmacokinetics

(a) Aging and the distribution and clearance of drugs in general

(b) Aging and the pharmacokinetics of anaesthetics

Shock and the pharmacokinetics of anaesthetics

Cimetidine interactions in anaesthesia

Conclusion

\section{Introduction}

\section{(a) Background}

A major component of the practice of anaesthesia is the administration of drugs to produce the pharmacodynamic endpoints of unconsciousness, analgesia and skeletal muscle relaxation while maintaining haemodynamic stability and adequate ventilation during surgery. To produce these effects a host of drugs may be given, either intermittently or continuously, intravenously or by inhalation. The pharmacodynamic effects of each of the drugs depend in part on the dose administered and on the pharmacokinetics of the drug, that is on all the processes which determine the concentration of the drug at any time both in blood and at the site of action. Many factors influence the pharmacokinetics of drugs, e.g. physiologic status, age, obesity, pregnancy, or the co-administration of other drugs which alter the way the body handles the drug of interest. Any of these factors can alter the dose response relationship for anaesthetic agents.

Over the last 20 years there has been a dramatic increase in pharmacokinetic research. This increase has resulted in part from improvements in analytical techniques for the measurement of drugs and metabolites, from the use of the computer for mathematical modelling, and from the realization of the importance of pharmacokinetic information for optinizing therapy and preventing toxicity. However, a deterrent to studying pharmacokinetics for many practitioners is the rigorous mathematical analysis often used in pharmacokinetic studies. In this article we will use words rather than mathematics to describe key pharmacokinetic principles. With thiopental as an example, the effects of aging and shock on pharmacokinetics will be examined. Finaliy, we will illustrate the problem of drug interactions producing pharmacokinetic changes in patients on chronic cimetidine therapy.

\section{(b) History of Pharmacokinetics}

Since the time of Paracelsus in the 16th century, it has been recognized that there is a causal relationship between the dose of a drug and the therapeutic or toxic effect. The measurement of drugs in blood was first attempted using alcohol by students of Rudolph Bucheim at the University of Dorpat in the 19th century. In 1866, one of his students, Oswald Schmiedeberg, submitted his dissertation in fulfillment of the requirements for the degree of Doctor of Medicine on the subject of an analytical method for the determination of the anaesthetic chloroform in blood.

Mathematics was first applied to dose-concentration-activity data by Widmark in 1919 and his approach was expanded in two theoretical review articles on kinetics published by Tcorell in $1938 .^{2}$ The term pharmacokinetics was coined by Dost ${ }^{2}$ to mean the science of quantitative analysis between an organism and a drug. The first review in the English language of the state of knowledge in pharmacokinetics was published by Nelson in $1961 .^{3}$ Since that time, the study of pharmaco-

From the Department of Anaesthesia, Queen's University, and Hotel Dieu Hospital, Kingston, Ontario

Address correspondence to: Dr. B. Milne, Department of Anaesthesia, Hotel Dieu Hospital, Kingston, Ontario, K7L 3H6.

This work was supported in part by a grant from Associated Medical Services. 
kinetics has expanded greatly and has been the subject of several review articles and books. ${ }^{4-7}$

Pharmacokinetics involves the application of mathematical models to drug concentration-time data to obtain rate and volume estimates for movement between and size of abstract body compirtments. Recognizing the limitations and dealing only in abstraction, in recent years the area of physiological pharmacokinetics has been developed. This approach differs from classic pharmacokinetics by using known organ size and blood flows instead of abstract compartments in mathematical modelling.

The concentration of a drug in blood is of interest as an indicator of the drug concentration at the site of action, since it is the latter which determines the ultimate drug effect. However, the relationship between the blood and tissue concentrations may not be absolute. Indeed for some drugs (e.g. narcotics) a notable dissociation between concentrations measured and blood and pharmacodynamic effect (analgesia) has been reported. Unfortunately, techniques for the quantitative determination of clinical drug effects have developed more slowly than techniques for measurement of drug concentrations. As a result data from advanced analytical methods too often are correlated with data from relatively crude clinical measurements with poorly controlled reproducibility. ${ }^{8}$ With improvement in quantitative clinical techniques and recognition of the importance of coupling pharmacokinetics with a pharmacodynamic endpoint, in future a more balanced perspective of the pharmacology of drugs will be achieved..$^{9,10}$

\section{Definitions}

Most anaesthetic agents are administered by either internittent intravenous push (bolus), or by inhalation. In this article we will focus on principles relating to intravenous agents.

FIRST ORDER REACTION - reaction in which the rate of change in drug amount is proportional to the concentration of the drug. Thus, the higher the drug concentration in a compartment the more drug leaving that compartment in a given time. For most drugs at usual therapeutic concentrations, elimination from blood proceeds in a first order process with a constant percentage of the drug concentration present in blood eliminated per unit time, e.g. morphine, thiopental.
ZERO ORDER REACTION - reaction that proceeds at a constant rate independent of the drug concentration such that a constant amount of drug is eliminated per unit time. The most commonly cited example of a drug eliminated by zero order processes is alcohol. It should be noted that for several drugs the administration of high doses may result in saturation of the drug metabolizing enzymes and a transition from first to zero order elimination. Thus, the rate of elimination is reduced and if drug dosing continues toxic levels may be reached. This transition from first to zero order kinetics has been reported for aspirin and thiopental but it is postulated that this may occur for any drug if a high enough blood concentration is reached.

STEADY STATE - dynamic equilibrium e.g. when intake of drug is equal to elimination and the amount in the body remains constant. Since intravenous anaesthetics are given by intermittent bolus at time intervals dictated by the pharmacodynamic endpoints of anaesthesia and haemodynamic stability it is unlikely that pharmacokinetic steady state is achieved for these agents.

HALF-LIFE $\left(t_{1 / 2}\right)$ - the time required for the concentration of the drug in plasma to fall by 50 per cent. Half-life has meaning only for drugs showing first order elimination since for zero order processes a constant amount is eliminated regardless of the concentration. For drugs given by IV bolus and showing first order elimination, the concentration initially falls very rapidly. This is the distributive or alpha $(\alpha)$ phase and although a $t_{1 / 2}\left(t_{1 / 2} \alpha\right)$ can be calculated, this $t_{1 / 2} \alpha$ is of limited usefulness. Subsequently the concentration falls more slowly; this is the elimination (Beta, $\beta$ ) phase and the $t_{1 / 2} \beta$ calculated is the most important $t_{1 / 2}$ estimate and is most commonly referred to simply as the "halflife."

APPARENT VOLUME OF DISTRIBUTION (Vd) - is an estimate of the extent of the distribution of the drug through body fluids and uptake by tissues. A large apparent volume of distribution implies wide drug distribution or tissue uptake or both. For some drugs, the volume of distribution may exceed the actual body volume, indicating that the concentration in some tissues must be considerably higher than the concentration in blood. Conversely, a very small volume of distribution implies that the dng is 
retained to a large extent within the blood and tissue uptake is limited.

ELIMINATION - sum of metabolic and excretory processes causing disappearance of pharmacologically active drugs from body fluids. Hepatic biotransformation, excretion by the kidney, exhalation by lungs and faecal excretion are the usual methods of drug elimination. In pharmacokinetic analysis drug elimination from the central compartment is best described by the clearance of the drug.

CLEARANCE - is a very important pharmacokinetic concept. Clearance is a direct index of elimination from the central compartment and is expressed in millilitres per minute. Clearance is inversely proportional to elimination $t_{1 / 2}$ (short $t_{1 / 2}$, rapid clearance), and directly proportional to the apparent volume of distribution (at any $t_{1 / 2}$, the larger the volume of distribution (Vd) the greater the clearance). This relationship is best expressed as follows:

Clearance $=0.693 \times \mathrm{Vd}$

FRST PASS EFFECT - a phenomenon characterized by drug uptake and elimination in the liver during the first passage of drug into the circulation after administration by a hepatic route, i.e. orally, intraperitoneally or via the splenic or portal veins such that all access to the peripheral venous system is by way of the hepatic portal system. As a result of the first pass effect, a significant fraction of the administered dose does not reach the systemic circulation. The factor which determines the first pass effect is the ability of the liver to extract the drug as it perfuses through the hepatic capillaries. This is likely a reflection of the overall activity of the drug-metabolizing enzymes or other rate-limiting processes involved in elimination. Propranolol is an example of a drug which undergoes extensive first pass elimination. Since most drugs of interest in anaesthesia are not administered by hepatic routes this concept is of less interest than the more general concept of hepatic clearance.

HEPATIC CLEARANCE - refers to the volume of blood flowing to the liver from which drug is completely removed in unit time. Hepatic clearance is a function of liver bload flow (in normals, approximately 25 per cent of the cardiac output), and the ability of the organ to extract the drug (as described above, a function of the metabolic activity of the liver for the drug of interest). Both of these factors may change, and the effect of these changes will vary depending upon the particular characteristics of the drug of intcrest. For example, if the liver blood flow is reduced, with no change in enzymatic activity, a drug which has a very large hepatic clearance will be cleared much less rapidly, and toxicity may result. However, little change will be seen for a drug which has a small hepatic clearance. Conversely, if the ability of the liver to metabolize a drug is doubled, a more marked increase in clearance may be seen for a drug for which liver metabolism is initially small than for drugs which already show a very marked hepatic extraction.

ONE-, TWO-, THREE-COMPARTMENT MODELS - the models used in pharmacokinetics vary in complexity. The simplest, a one-compartment model, assumes that drugs are distributed instantly and homogeneously through the fluids and tissues of the body. Although appealing because of simplicity, the model does not account accurately for the true time course of most drugs in the body. A twocompartment open model assumes that the drug is distributed between a central compartment and a peripheral compartment. This model is used most frequently in kinetics and is usually satisfactory. However, for some drugs more complicated analysis is required and three or more compartments may be invoked. It should be noted that in classic pharmacokinetics these are abstract compartments and do not describe any particular body organ. With the two-compartment model, the central compartment is usually considered as the blood plus those organs which receive a major percentage of the cardiac output, such as the brain, heart, liver. The peripheral compartment generally refers to tissue with a more limited rate of perfusion.

\section{Aging}

(a) Aging and the distribution and clearance of drugs in general

Many changes in body function occur with age. The extent that the pharmacokinetics of a drug changes depends on the magnitude of change in organ systems with age and on the specific factors responsible for the distribution and clearance of each drug.

With age, changes in body composition, cardiac 
output and blood flow occur and can markedly alter the distribution of drugs. ${ }^{11,12}$ The ratio of fat to lean mass doubles between the ages of 25 and 75 and results in an increase in volume of distribution of lipophilic drugs. The concentration of plasma albumin declines by 20 per cent by the age of 65 , resulting in an increase in the free fraction of highly bound drugs. Since it is the free drug which is in equilibrium with tissues, an increase in the free fraction can result in a greater drug effect in the elderly following a standard adult dose. Cardiac output declines by one per cent per year after the age of 25 and there is a yearly decline in blood flow to the brain $(0.35-0.5$ per cent), liver $0.3-1.5$ per cent) and kidney (1.1-1.9 per cent). ${ }^{11,12}$ Since the decrease in flow is not uniform to all tissues an age-related change in drug distribution secondary to flow alterations can be anticipated.

The biotransformation and clearance of drugs can change with age. This change may be due in part to the decline in blood flow to the liver, a major organ of metabolism. The reduced blood flow results in a decrease in the removal of drugs such as lidocaine and meperidine which are highly extracted by the liver. ${ }^{13}$ Functional changes in the liver with age can also result in a decrease in drug metabolism. In rats, the basal activity of several microsomal enzymes (e.g. hexobarbital hydrolase, aminopyrine $\mathrm{N}$ demethylase) has been shown to decline markedly (45 per cent) with age. ${ }^{11,14}$ This can result in a marked prolongation in the half life of drugs metabolised in the liver. For example in man the half life of amobarbital has been shown to increase from 22.8 hours in the young to 86.6 hours in the elderly, a change attributed to the decrease in liver metabolism of the drug. ${ }^{15,16}$

The kidney is also an important organ for drug metabolism and clearance. A reduction in clearance by the kidney with age may result from the age-related decrease in kidney blood flow of 45-50 per cent by 65 years of age. ${ }^{11,12}$ The functional loss in the kidney with age may also be a factor. Between the ages of 20 and 80 there is a 20 per cent decrease in kidney size and a 30 per cent loss of functional glomeruli. ${ }^{13}$ Thus the plasma half lives of drugs such as digoxin and penicillin, for which kidney clearance is important, are prolonged in the gerialric patient. ${ }^{11}$

(b) Aging and pharmacokinetics of anaesthesia Age-related changes in the phamacokinetics of some anaesthetics have been reported. A comparison of the plasma concentration of the analgesic meperidine following a single $1.5 \mathrm{mg} \cdot \mathrm{kg}$ dose to a group of young (under 30) and old (over 70) subjects revealed that the plasma levels in the old subjects were more than twice as high as in the young for more than seven hours after the drug was administered. ${ }^{17}$ This difference has been attributed to changes in binding and clearance of meperidine with age. ${ }^{17,18}$ In a study comparing the pharmacokinetics of diazepam following a single intravenous dose in young and old men and women, many parameters were found to change. ${ }^{19}$ For women, clearance was the same in the young and old, but for men the clearance in the young was greater than in the elderly. The volume of distribution of diazepam became larger with age while protein binding decreased. ${ }^{19}$ With pancuronium, a neuromuscular blocking agent, no change in dose requirement has been found with age in a study of 22 patients, suggesting no change in pharmacodynamics. However, the paralysis produced was found to be prolonged in the elderly, an observation attributed to reduced clearance of pancuronium in this population. ${ }^{20}$ These results are at variance, however, with an earlier study with 13 patients in whom no difference in the recovery time after pancuronium was observed with age. ${ }^{21}$ For thiopental, an increase in both $\mathrm{Vd}$ and $\mathrm{t}_{1 / 2}$ were found with age in 22 lean women undergoing abdominal surgery. In addition a significant correlation was found between age and the free or unbound fraction of drug. Although no assessment of sensitivity or recovery time was made, the authors suggested that due to the prolonged elimination of thiopental in the elderly this drug should be used cautiously in the geriatric population. $^{22}$

\section{Shock and pharmacokinetics of angesthetics}

Shock is defined as a state in which there is widespread, serious reduction of tissue perfusion which if prolonged leads to a generalized impairment of cellular function. ${ }^{23}$ While there are many clinical causes of shock the most common are hypovolemia either due to external fluid loss or internal sequestration, or cardiogenic shock following myocardial infarction or secondary to severe rhythm disturbances of congestive cardiac failure. Although in preparing to anaesthetize a patient in shock initial measures are taken to improve cardiovascular function, such as the administration of 
fluids and inotropic agents, a low output state may persist at the time of anaesthesia. Thus, the effect of shock on the pharmacokinetics of anaesthetics is of concern to the anaesthetist

One of the first reports alluding to a change in the response to anaesthetics in shock was that of Halford in 1943 describing the use of thiopental in anaesthetizing sailors wounded at Pearl Harbor. ${ }^{24}$ In this report, thiopental was administered in large doses $(0.5 \mathrm{gm})$ as the sole anaesthetic agent, and in many instances respiratory failure and death rapidly ensued. The author concluded that intravenous anaesthesia was an excellent form of euthanasia and that open drop ether was a safer anaesthetic technique.

At therapeutic concentrations, thiopental is highly bound ( 86 per cent) to plasma proteins. ${ }^{25}$ Following intravenous administration there is a very rapid initial decay in concentration, with a much slower elimination phase $\left(t_{1 / 2} \cong 6.2\right.$ hours). ${ }^{26}$ The rapid onset of action results from the rapid distribution of thiopental into the brain as a result of the high cerebral blood flow, the lipid solubility of the drug and the easy transit of thiopental across the blood brain barrier.

In shock and other low cardiac output states there are several haemodynamic changes which alter the pharmacokinetics of thiopental. The extent of the change will depend on the degree and duration of the shock state and the other concomitant problems such as reduction in liver blood flow or liver damage. ${ }^{27,28}$ In general, in any individual hypotension can be induced by the injection of thiopental. ${ }^{29}$ The factors which determine the degree of cardiovascular depression include: (1) dose of drug, (2) speed of injection, and (3) volume of the central compartment. With respect to speed of injection, with a rapid injection there is less protein binding initially and more drug available to highly perfused tissue such as brain and heart, and a greater sedative and hypotensive cffect. With respect to volume, in shock states particularly following haemorrhage, the central volume into which the drug is injected is reduced. As a result the initial concentration is higher and a greater distribution into brain and heart may occur with a resultant marked sedation and hypotension. In the sailors at Pearl Harbor, the injection of thiopental into a markedly reduced central volume secondary to haemornhage probably caused elevated concentrations, respiratory failure and death.
In addition to a more rapid sedation and marked hypotension with thiopental in low output states, other haemodynamic changes can alter the rate of elimination of the drug. For example, thiopental is eliminated by hepatic biotransformation by the liver mixed function oxidase enzyme. In shock, studies in dogs have shown a reduction in liver blood flow and prolongation of the $t_{1 / 2}$ of indocyanine green, a dye which undergoes extensive first-pass clearance ${ }^{30}$ Studies in patients with cardiac failure have shown a reduction in liver blood flow and metabolism with a resultant reduction in lidocaine clearance. ${ }^{31}$ With thiopental, although no data are available one would expect that the volume of distribution and clearance would be reduced and the $t_{1 / 2}$ prolonged. As a result, the patient in shock would likely be more responsive than the normal patient at low thiopental induction doses and also recover more slowly following anaesthesia.

These changes which are predicted for thiopental have been described in some detail for lidocaine, a drug extensively cleared by the liver. After intravenous administration, the volume of distribution and clearance of lidocaine are reduced in experimental animals in shock and in patients in congestive cardiac failure. ${ }^{31}$ Thus, initial concentrations in well-perfused tissue such as brain and heart will be high since perfusion of these organs is preserved in all but the most severe states, while clearance of the drug is impaired. Both central nervous system and cardiac toxicity may result if care is not taken to give reduced doses. Due to the variability in the clinical picture of shock, with great variation in the haemodynamic profile and magnitude of effect on phannacokinetics, the determination of drug concentrations in blood can be a valuable adjunct in therapy in low output states. ${ }^{29}$

\section{Cimetidine interactions in anaesthesia}

Another potential problem to be considered with respect to pharmacokinetics and anaesthesia is that of drug interactions. The average patient may receive eight different medications during hospitalization plus another five to ten drugs during anaesthesia. Drugs may interact with anaesthetic agents by: direct chemical interaction (mixing of meperi-

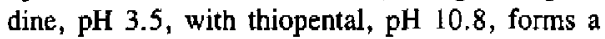
precipitate), interaction at the site of absorption (vasoconstriction by epinephrine will delay the uptake of a local anaesthetic), alteration of protein binding (protein-bound barbiturates are displaced 
by $x$-ray contrast media), competition for receptor sites (reversal of d-tubocurarine block by neostigmine), altering metabolism (echothiopate depletes plasma cholinesterase and prolongs the action of succinylcholine) and altering excretion (increased excretion of phenobarbital with alkalinization of the urine). ${ }^{33}$

Anaesthetists are dealing with patients who are taking an ever-increasing variety of medications which may interact with anaesthetic agents. One such drug is cimetidine and after first describing the general pharmacology of the drug a hypothetical case of drug interaction during anaesthesia in a patient receiving cimetidine will be discussed.

Cimetidine, an $\mathrm{H}_{2}$ receptor antagonist, is one of the most frequently prescribed medications today, rivalling diazepam. It is widely used in the treatment of peptic ulcer and prior to surgery to increase gastric $\mathrm{pH}$ and decrease the volume of gastric contents. Due to the diffuse location of histamine receptors, cimetidine alone has many side effects which may concem the anaesthetist. Histamine enhances cardiac automaticity and this effect may be antagonized by $\mathrm{H}_{2}$ receptor antagonists. This effect may account for the dysrythmias, bradycardia, hypotension and cardiac arrest which have been reported following intravenous cimetidine. Histamine receptors are distributed in many brain areas and neural dysfunction with confusion, agitation and coma has occurred with cimetidine. In addition, cimetidine has been reported to cause haematological abnormalities, bone marrow suppression, a weak antiandrogenic effect, and elevation of transaminase, alkaline phosphatase and serum creatinine levels. ${ }^{33}$

Cimetidine also affects liver function by altering liver metabolism and decreasing liver blood flow, and this may result in problems during anaesthesia. Its effect on metabolism is secondary to binding of cytochrome $\mathrm{p} 450$ and inhibition of drug metabolism while the action on $\mathrm{H}_{2}$ receptors in blood vessels results in a reduction in liver blood flow. This potent inhibition of hepatic drug metabolizing enzymes reduces the clearance of many drugs, e.g. warfarin, diazepam and chlordiazepoxide, and the decrease in liver blood flow increases the bioavailability of drugs with high hepatic extraction ratios such as lidocaine, morphine, and propranolol..$^{34-36}$

Consider a hypothetical elderly female patient whose problems include a bleeding duodenal ulcer for which she had been on oral cimetidine and is now receiving the drug intravenously, and reactive airway disease, for which she is receiving a theophylline preparation. With such a patient presenting for surgery a number of things must be kept in mind with regard to her cimetidine therapy. Preoperatively or during the induction of anaesthesia, diazepam may be administered. Increased sedation or prolonged postoperative sedation due to decreased metabolism by cimetidine may result. This may be expected because diazepan and chlordiazepoxide are demethylated to active metabolites by the cytochrome p450 enzyme system before glucuronidation to inactive compounds. Since oxazepam and lorazepam undergo initial glucuronidation and do not possess active metabolites ${ }^{35,36}$ it would be better to use these agents or decrease the dose of diazepam administered. It should also be noted that intravenous cimetidine increases the absorption of oral diazepam and lorazepam probably due to alteration of gastric acidity. ${ }^{38}$ Metabolism of warfarin and anticonvulsants such as carbamazepine and phenytoin is also affected by cimetidine and a potentially lethal interaction causing respiratory depression between morphine and cimetidine has been reported. ${ }^{35,39}$

Clearance of drugs with high hepatic extraction ratios such as propranolol, metopolol, labetalol and lidocaine is reduced by cimetidine. For example if this patient began to have premature ventricular contractions requiring treatment with lidocaine, or if lidocaine were to be used as a local anaesthetic one would have to take her cimetidine therapy into account. First, it would be prudent to monitor serum theophylline levels as these premature ventricular contractions may have arisen from a heart which was made irritable by theophylline. Cimetidine significantly alters theophylline clearance and elimination $t_{1 / 2}$ after both acute and chronic administration, probably by inhibiting the conversion of theophylline to 3-methylxanthine. Patients on cimetidine with initial theophylline concentrations $>15 \mu \mathrm{g} \cdot \mathrm{ml}$ or those in whom its clearance is reduced (e.g. the elderly) will require a theophylline dose reduction of approximately $30-50$ per cent. $^{35,40}$

Cimetidine results in increased toxicity when repeated boluses or infusions of lidocaine are administered. DiFazio reported a 38 per cent increase in plasma $t_{1 / 2}$ and a decrease in total body clearance of lidocaine in rats treated with cimetidine. ${ }^{41}$ Increased toxicity and reduced clearance of 
lidocaine by cimetidine has been reported in $\operatorname{man}^{42}$ and it has been suggested that a number of arrhythmic episodes and unexplained deaths in coronary care units may be the result of lidocaine-cimetidine interaction. ${ }^{43}$ Thus, if a lidocaine infusion were used to treat arrhythmias in this patient a reduced rate should be used.

Cimetidine may also have other deleterious effects. Emergence delirium could be compounded by cimetidine due to its effect on histamine receptors in the CNS. Since cimetidine decreases liver blood flow, an increase in liver toxicity caused by some of the halogenated anaesthetic agents could perhaps be anticipated. Care should be taken not only in patients on chronic therapy but in those receiving the drug acutely as cimetidine may produce changes in drug elimination as quickly as one day after its introduction to a treatment regimen. Toxicity may be minimized by being aware of potential drug interactions, monitoring the patient for adverse drug effects with appropriate tests (e.g. concentrations of the drug if available), lowering the dose of the drug suspected of interacting, or switching to another drug. ${ }^{44}$

\section{Conclusion}

Pharmacokinetics is a branch of pharmacology concerned with the effect of the organism on drugs and is one of the factors which determine the magnitude of the drug effect. In normals considerable variation is seen in the pharmacokinetics of drugs. Changes in the pharmacokinetics of anaesthetics have been documented under many circumlstances including in the elderly, in patients in shock, and in patients on other drug therapy such as cimetidine. These alterations should be considered during the practice of anaesthesia in order to avoid predictable toxicity due to excessive drug concentrations or reduced elimination.

Over the past 20 years much information has been reported conceming both the pharmacokinetics and pharmacodynamics of drugs, but these determinations have frequently been made independently of each other. In future research, the combination of pharmacokinetics with a pharmacodynamic endpoint will provide a more comprchensive approach to the pharmacology of drugs and permit more rational drug therapy.

\section{Acknowiedgement}

The authors would like to thank Ms. Mary Burgstaller for her assistance in the preparation of this manuscript.

\section{References}

1 Buter TC. Some historical reflections on drug metabolism. Ann NY Acad Sci 1971; 179: 502-7.

2 From Wagner JG. Fundamentals of clinical pharmacokinetics. Drug Intelligence Publications Inc., Hamilton, Illinois, 1975

3. Nelson $E$. Kinetics of drug absorption, distribution, metabolism and excretion. J Pharm Sci 1961; 50: 181-92.

4 Greenblat DJ, Koch-Weser J. Clinical Pharmacokinetics. N Engl J Med 1975; 293: 702-5, 964-70.

5 Gibaldi M, Perrier D. Pharmacokinetics. Marcel Dekker Inc. New York, 1975.

6 van Rossum JM (ed). Kinetics of Drug Action Handb. Exp. Pharm. 471977.

7 Stanski $D R$, Watkins WD. Drug disposition in Anesthesia. Grune \& Stratton, New York, 1982.

8 Boreus $L O$. Pharmacokinetics and clinical response. Eur J Clin Pharmacol 1980; 18: 51-3.

9 Dollery CT. Phamacokinetics - master or servant. Eur J Clin Pharmacol 1973; 6: 1-2.

10 Turner $P$. Blood level or pharmacological response. BI J Clin Pharnacol 1974; I: 11.

If Schmucker DL. Age-related changes in drug distribution. Pharmacological Reviews 1979; 30: 44556.

12 Ritschel WA. Drug action and interaction in the geriatric paticnt. Sonderdruck Scientia Pharmaceutica 1977; 45: 304-10.

13 Schumacher GE. Using pharmacokinetics in drug therapy VII. Pharmacokinetic factors influencing drug therapy in the aged. Am J Hosp Pharm 1980; 37: 559-62.

14 Kato $R$, Vassanelli P. Frontino G, Chiesera E. Variation in the activity of liver microsomal drug metabolizing enzymes in rat in relation to the age. Biochem Pharmacol 1964; 13: 1037-51.

15 Irvine RE, Grove J, Toseland PA, Trounce JR. The effect of age on the hydroxylation of amylobarbitonc sodium in man. Br J Clin Pharmacol 1974; 1: 41-3.

16 Ritschel WA. Age-dependent disposition of amobarbital: Analog computer evaluation. J Am Ger Soc 1978; 26: 540-3. 
17 Clan K, Kendall MJ, Mitchard M, Wells WDE. Effect of ageing on plasma pethidine concentrations. Br J Clin Pharmacol 1975; 2: 297-302.

18 Mather LE, Meffin PJ. Clinical pharmacokinetics pethedine. Clin Pharmacokinetics 1978; 3: 352-68.

19 Greenblatt DJ, Allen MD, Harmatz JS, Shader Rl. Diazepam disposition determinants. Clin Pharmacol Ther 1980; 27: 301-12.

20 Dupaldestin P, Berger JL, Saada J, Desmonts $M D$. Pancuronium: pharmacokinetics and pharmacodynamics in the elderly. Anesthesiology 1980; \$3: $\$ 284$

21 Chmielewski AT, Pybus DA, Loach AB, Goat $V A$. Recovery from neuromuscular blockade: a comparison betwcen old and young patients. Anaesthesia 1978; 33: 539-42.

22 Jiang D, Mayersohn $M$, Perrier D, Calkes $S J$, Saunders $R$. Thiopental disposition as a function of age in female patients undergoing surgery. Anesthesiology 1982; 56: 263-8.

23 Harrison's Principles of Internal Medicine 9th edition. Editors: Isselbacher KJ, Adams RD, Braunwald E, Petersdorf RG, Wilson ID. McGrawHill Book Company, New York 1980.

24 Halford RJ. A critique of intravenous anaesthesia in war surgery. Anesthesiology 1943; 4: 67-9.

25 Becker KE. Gas chromatographic assay for free and total plasma levels of thiopental. Anesthesiology 1976 ; 45 ; 656-60.

26 Ghoneim MM, Van Hamme MJ. Pharmacokinetics of thiopentone: Effects of enflurane and nitrous oxide anaesthesia and surgery. Br J Anaesthesia 1978; 50: 1237-41.

27 Pardy $B J$, Dudley $H A F$. Sequential patterns of hemodynamic and metabolic changes in experimental hypovolemic shock. 1. Response to acute hemorrhage. Br J Surg 1979; 66; 84-8.

28 Birgens HS, Henrikson J, Matzen P, Poulsen H. The shock liver. Acta Med Scand 1978; 204: 417-21.

29 Duvaldestin $P$. Pharmacokinetics in intravenous anaesthetic practice. Clin Pharmacokinet 1981;6 [1-82.

30 Nxumalo $J L$, Teranaka $M$, Schenk WG. Sensitivity of indocyanine green half-life changes relative to sirculatory shock state. J Surg Res 1977; 23:400-4.

31 Benowitz NL, Meister W. Pharmacokinetics in patients with cardiac failure. Clin Pharmacokinet 1976; 1: 389-405.
32 Cullen $B F$, Miller, MG. Drug interactions and anesthesia: A Review. Anesth Analg 1979; 58: 413-23.

33 Manchikanti L, Kraus JW, Edds SP. Cimetidine and related drugs in anesthesia. Anesth Analg 1982; 61: 595-608.

34 Somogyi A, Gugler R. Drug interaction with cimetidine. Clin Pharmacokinet 1982; 7: 23-41.

35 Bauman JH, Kimelblatt BJ. Cimetidine as an inhibition of drug metabolism: Therapeutic implications and review of the literature. Drug Intell Clin Pharm 1982; 16: 380-6.

36 Feely $J$, Wilkinson $G R$, Wood AJJ. Reduction of liver blood flow and propranolol metabolism by cimetidine. N Engl J Med 1981; 304: 692-5.

37 Klotz V, Reiman I. Delayed clearance of diazepam due to cimetidine. N Engl J Med 1980; 302: 1012-4.

38 McGowan WAW, Dundee JW. The effect of intrayenous cimetidine on the absorption of orally administered diazepam and lorazepam. Br J Clin Pharmacol 1982; 14: 207-11.

39 Fine A, Churchill DN. Potentially lethal interaction of cimetidine and morphine. Can Med Assoc J 1981; 124: 1434-6.

40 Campbell MA, Plachetka JR, Jackson JE, Moon $J F$, Finley $P N$. Cimetidine decreases theophylline clearance. Ann Intern Med 1981; 95: 68-9.

41 Difazio CA, Moscicki JC, DiFazio CJ. Cimetidjne inhibits lidocaine plasma clearance. Anesthesiology 1982; 57: A188.

42 Feely J, Wilkinson GR, McAllister CB, Wood $A J J$. Increased toxicity and reduced clearance of lidocaine by cimetidine. Anm Intern Med 1982; 96: 592-4.

43 Lidocaine-cimetidine interaction can be toxic. JAMA 1982; 247: 174-5.

44 Bauman JH, Kimelbiat BJ, Wible DA, Kucich $J M$. Cimetidine and drug metabolism. Can Med Assoc J 1982; 127: 199. 